\title{
Evaluating a SLAM-based Handheld Augmented Reality Guidance System
}

\author{
Jarkko Polvi ${ }^{1}$, Takafumi Taketomi ${ }^{1}$, Goshiro Yamamoto ${ }^{1}$, Mark Billinghurst ${ }^{2}$, Christian Sandor $^{1}$, \\ Hirokazu Kato ${ }^{1}$ \\ ${ }^{1}$ Nara Institute of Science and Technology, Japan \\ \{jarkko-p, takafumi-t, goshiro, sandor, kato\}@is.naist.jp \\ ${ }^{2}$ University of Canterbury, New Zealand \\ mark.billinghurst@canterbury.ac.nz
}

\begin{abstract}
In this poster we present the design and evaluation of a Handheld Augmented Reality (HAR) prototype system for guidance. We have evaluated our prototype in two generic guidance scenarios: a device set-up and an object assembly.
\end{abstract}

\section{Categories and Subject Descriptors}

H.5.2 [User Interface]: Graphic user interface (GUI), Screen design, user-centered design.

\section{Keywords}

Handheld augmented reality; guidance; user evaluation.

\section{INTRODUCTION}

HAR has a huge potential to introduce Augmented Reality (AR) to the mass consumer market due to widespread use of suitable handheld devices. Compared to other mobile AR mediums, HAR also offers superior information input methods and a possibility for easier collaboration. However as Olsson et al. [1] and Grubert et al. [2] point out, HAR is not currently considered useful due to, for example, insufficient utility and irrelevant content. Thus, it is important to evaluate the usefulness of HAR in different kinds of practical use scenarios.

We are developing HAR for practical use and in this poster, we focus on the usefulness of HAR in two generic guidance scenarios. We describe the design and evaluation of our prototype HAR guidance system and address its usefulness and usability related issues. The contribution of our work is the lessons learned from the evaluation of the prototype system.

\section{PROTOTYPE SYSTEM}

The prototype system was developed for the iPad and it uses Simultaneous Localization And Mapping (SLAM) for tracking. Markerless SLAM tracking allows the system to be used more freely in different kinds of unknown environments. The task flow in our system is as follows: First the desired area of interest (a SLAM map) needs to be selected from an overview image of the environment. After the area is selected the SLAM map tracking needs to be initialized by choosing the correct viewpoint. Finally, the annotations (short 2D textual messages) can be seen overlaid onto the real world. The system also enables AR content authoring, but in this work we focus only on the guidance.

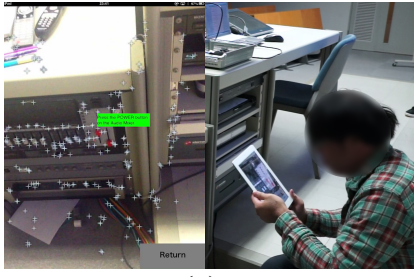

(a)

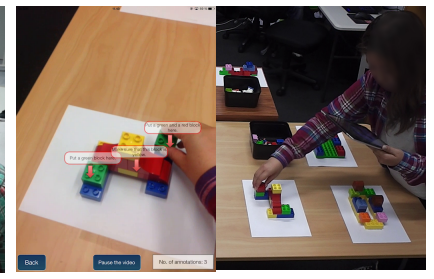

(b)
Figure 1: The evaluation scenarios: the device set-up scenario (a) and the object assembly scenario (b).

\section{USER STUDIES}

We have performed two user studies on our prototype system. A small-scale evaluation (6 participants) was conducted on the initial prototype in a device set-up scenario (Fig. 1a) in order to identify usability issues. We improved the system and then conducted a within-group comparative study (27 participants) against two iPad multimedia guides: a picture and a video guide. This study was done in an object assembly scenario (Fig. 1b) and we measured performance and subjective feedback.

We derived five guidelines based on the results of our evaluations: location of the AR environment, the information about the offscreen AR content, navigational shortcuts, view pausing, and feedback. We were unable to find performance benefits from the use of AR compared to conventional multimedia guides. Main reasons for this was the overall complexity of the AR compared to the two other guides and the simplicity of the assembly tasks.

\section{CONCLUSION}

We have described the design and evaluation of our HAR guidance prototype. Even though we were unable to prove the superiority of AR compared to conventional guides, we gained valuable information about the possible improvement areas. HAR could be beneficial in more complex guidance tasks that require, for example, several viewing angles. The use of HAR in generic guidance could be justified if other benefits of HAR (information input, collaboration etc.) are also made use of. Future work will see additional improvements made to our prototype system based on the results of the comparative evaluation. We will then expand our system to more complex practical scenarios that require authoring AR content and inputting different kinds information.

\section{REFERENCES}

[1] T. Olsson and M. Salo. Online user survey on current mobile augmented reality applications. In Proceedings of International Symposium on Mixed and Augmented Reality, pp. 75-84, 2011.

[2] J. Grubert, T. Langlotz, R. Grasset. Augmented reality browser survey. Graz University of Technology, 2011. 